\title{
Síndrome de Klinefelter como causa de úlcera crónica de difícil manejo. Reporte de caso
}

\section{Klinefleter syndrome as a cause of difficult chronic ulcer managment. Case report}

\author{
Melissa Espinosa-Flores ${ }^{1 *}$, J. Fernando Naranjo-Saltos ${ }^{1}$, Cristian J. Unda-Proaño ${ }^{1}$ y Ana G. Medina-Torres ${ }^{2}$
}

'Departamento de Medicina Interna, Hospital Eugenio Espejo, Quito, Ecuador; ${ }^{2}$ Departamento de Anestesiología, Hospital

Metropolitano "Dr. Bernardo Sepúlveda", Nuevo León, México

\section{RESUMEN}

El síndrome de Klinefelter es una enfermedad congénita única del sexo masculino. Se manifiesta clínicamente con un fenotipo ginecoide, alteraciones psicosociales, neurológicas, hormonales, endocrino-metabólicas y sexuales. La presencia de úlceras en miembros inferiores es una complicación no muy reconocida en este síndrome, su patogénesis se asocia con anomalías de la fibrinólisis y estados protrombóticos. Presentamos el caso de un paciente de sexo masculino con úlcera crónica de pierna izquierda de 21 años de evolución, quien en análisis cromosómico mostró un cariotipo $47 \mathrm{XXY}$, compatible con síndrome de Klinefelter.

Palabras clave: Síndrome de Klinefelter. Úlceras crónicas. Testosterona.

\begin{abstract}
Klinefelter syndrome is a male congenital disease. It manifests clinically with a gynecoid phenotype psychosocial, neurological, hormonal, endocrine-metabolic and sexual alterations. The presence of ulcers in the lower limbs is a not very well recognized complication in this syndrome, its pathogenesis is associated with anomalies of fibrinolysis and prothrombotic states. We present a case of a male patient with chronic left leg ulcer of 21 years of evolution who in chromosomal analysis showed a $47 \mathrm{XXY}$ karyotype compatible with Klinefelter syndrome.
\end{abstract}

Key words: Klinefelter syndrome. Chronic ulcers. Testosterone.

\section{Correspondencia:}

*Melissa Espinosa-Flores

E-mail: dra.melissaef@gmail.com
Fecha de recepción: 04-03-2021

Fecha de aceptación: 11-03-2021

DOI: 10.24875/RME.M21000009
Disponible en internet: 01-04-2021 Rev Mex Endocrinol Metab Nutr. 2021;8:143-7

2462-4144 / ๔ 2021 Sociedad Mexicana de Nutricion y Endocrinologia, AC. Publicado por Permanyer. Este es un artículo open access bajo la licencia CC BY-NC-ND (http://creativecommons.org/licenses/by-nc-nd/4.0/). 


\section{INTRODUCCIÓN}

En este reporte presentamos a un paciente con síndrome de Klinefelter que inicia su cuadro clínico con aparición de úlcera en miembro pélvico izquierdo no asociado a traumatismo o postración. El reto principal fue el adecuado control de la infección por medio de antibióticos y curaciones de manera continua, y finalmente, tomando en cuenta su patología de base, la administración de testosterona subcutánea como tratamiento final.

\section{CASO CLÍNICO}

Paciente de sexo masculino de 46 años, nacido y residente de las Ventanas (Los Ríos, Quito, Ecuador); divorciado, agricultor. Antecedentes de asma desde la niñez y alergia a las penicilinas. Fumador activo y bebedor de alcohol ocasional. Consumo crónico de naproxeno. Inicio de vida sexual a los 16 años.

El paciente refiere que desde hace 21 años cursa con lesión ulcerativa a nivel de miembro inferior izquierdo, que ha incrementado de tamaño a lo largo del tiempo. Se ha mantenido en observación y tratamiento en múltiples ocasiones sin obtener adecuada respuesta clínica.

Es ingresado a hospitalización por cuadro de dolor intenso en pierna izquierda, acompañado de dificultad para la deambulación y bipedestación. Durante su hospitalización se mantuvo en tratamiento antibiótico para una infección por Proteus mirabilis en cultivo de secreción con ciprofloxacino $500 \mathrm{mg}$ cada 12 horas por 12 días y clindamicina 800 mg cada 8 horas por 10 días; además se realiza limpieza quirúrgica, en donde se reporta tejido purulento y desvitalizado. Se interconsulta para la valoración de úlcera crónica de difícil manejo. Al examen físico, tensión arterial de 109/82 mmHg, frecuencia cardiaca de 72 latidos por minuto, frecuencia respiratoria de 18 respiraciones por minuto, afebril, con adecuada saturación al aire ambiente. Paciente con palidez generalizada, fenotipo gineocoide, estatura elevada, delgado, miembros
Tabla 1. Evaluación de paraclínicos

\begin{tabular}{lc}
\hline Exámenes de laboratorio & Valores de referencia \\
\hline Leucocitos & $6,610 \mathrm{~mm}^{3}$ \\
\hline Neutrófilos & $41.3 \%$ \\
\hline Linfocitos & $35.7 \%$ \\
\hline Hematocrito & $37.2 \%$ \\
\hline Filtrado glomerular MDRD & $64.13 \mathrm{ml} / \mathrm{min} / 1.73 \mathrm{~m}^{2}$ \\
\hline Colesterol total & $166 \mathrm{mg} / \mathrm{dl}$ \\
\hline LDL & $131 \mathrm{mg} / \mathrm{dl}$ \\
\hline Triglicéridos & $216 \mathrm{mg} / \mathrm{dl}$ \\
\hline Potasio & $5.91 \mathrm{mEq} / \mathrm{l}$ \\
\hline Hb glucosilada & $5.3 \%$ \\
\hline Proteínas totales & $7.21 \mathrm{~g} / \mathrm{dl}$ \\
\hline Albúmina & $3.59 \mathrm{~g} / \mathrm{dl}$ \\
\hline Globulina & $3.62 \mathrm{~g} / \mathrm{dl}$ \\
\hline Proteína en orina de $24 \mathrm{~h}$ & $248.60 \mathrm{mg} / \mathrm{dl}$ \\
\hline Pruebas hormonales & $18.10 \mathrm{ng} / \mathrm{ml}$ \\
\hline Prolactina & $19.9 \mathrm{U} / \mathrm{ml}$ \\
\hline FSH & $34 \mathrm{ng} / \mathrm{ml}$ \\
\hline Testosterona total & \\
\hline
\end{tabular}

MDRD: (ecuación) Modification of Diet in Renal Disease; LDL: lipoproteínas de baja densidad; FSH: hormona foliculoestimulante.

inferiores con mayor longitud que el tórax, cara redonda, ausencia de vello corporal. Cuello pequeño, cilíndrico. Tórax con presencia de ginecomastia derecha grado III e izquierda II. Abdomen con panículo adiposo de distribución centrípeta, presencia de equimosis infraumbilicales. Región genital con hipoplasia testicular, micropene. Extremidad pélvica izquierda con presencia de úlcera de $20 \mathrm{x}$ $16 \mathrm{~cm}$ en todo el diámetro de la pierna, que se extiende desde el tercio proximal hasta la región intermaleolar, de bordes irregulares, con zonas friables y tejido de granulación escaso, pulsos distales disminuidos en extremidad pélvica izquierda, se mantiene cubierta con productos de curación avanzada (Tabla 1).

\section{Estudios de imagen}

La ecografía testicular demuestra testículos pequeños, de estructura homogénea, derecho de $1.3 \times 1 \mathrm{~cm}$, epidídimo de $4 \mathrm{~mm}$; izquierdo de $1.2 \times 9 \mathrm{~mm}$ 
epidídimo de $4 \mathrm{~mm}$. Ecocardiograma: patología valvular aórtica orgánica, doble lesión con insuficiencia progresiva y estenosis leve; remodelado concéntrico del ventrículo izquierdo; fracción de eyección del ventrículo izquierdo $74 \%$ por Teich; insuficiencia mitral y tricuspídea leve. Insuficiencia pulmonar leve; presión sistólica de arteria pulmonar $35 \mathrm{mmHg}$; disfunción diastólica ligera con patrón de relajación prolongada tipo I del ventrículo izquierdo.

\section{DISCUSIÓN}

El síndrome de Klinefelter, descrito por primera vez en 1942 por Harry F. Klinefelter, es la anomalía congénita más común que causa hipogonadismo primario, ocurre en 1 de cada 1,000 nacidos vivos ${ }^{1}$. Los pacientes con este síndrome son hombres fenotípicamente, pero tienen al menos un cromosoma $X$ adicional, como resultado de la no disyunción de los cromosomas sexuales de cualquiera de los padres durante la división meiótica. Hormonalmente, los pacientes tienen niveles de testosterona de aproximadamente un $50 \%$ menos de lo normal debido a disgenesia del túbulo seminífero. Las consecuencias gonadales del síndrome incluyen escaso vello facial y corporal, testículos pequeños, disminución del recuento de espermatozoides e infertilidad $^{2}$.

El síndrome metabólico, que estaba presente en el caso descrito, es una complicación conocida del síndrome de Klinefelter, que se produce en hasta el $40 \%$ de los pacientes con esta patología ${ }^{3}$. El desarrollo del síndrome metabólico en estos pacientes se cree que es secundario al estado de hipogonadismo que conduce a la distribución de grasa del tronco, disminución de la masa muscular y la inactividad. Otras manifestaciones extragonadales del síndrome de Klinefelter incluyen anormalidades en los huesos largos con aumento de la longitud de las piernas; y hay algunos informes de anomalías psicosociales que llevan a interacciones sociales difíciles. Además, se han descrito asociaciones con venas varicosas, enfermedad tromboembólica, cáncer de mama y enfermedades autoinmunes, incluyendo lupus eritematoso sistémico 4 .
Las heridas que no sanan afectan a 6.5 millones de pacientes con un costo anual de 25 mil millones de dólares solo en los EE.UU. El diagnóstico diferencial en úlceras de extremidades inferiores está bien documentado en otros lugares, incluyendo la estasis venosa, enfermedad vascular periférica, diabetes, enfermedad autoinmune y estados protrombóti$\cos ^{5}$. La ulceración de las piernas es una complicación del síndrome de Klinefelter, con una prevalencia en miembros inferiores del 6-13\% ${ }^{6}$. La etiología de las úlceras en pacientes con síndrome de Klinefelter se cree que es multifactorial a causa de insuficiencia venosa crónica, obesidad, displasia arterial en las piernas y disminución de la fibrinólisis debido a los niveles elevados de inhibidor del activador del plasminógeno $1^{7}$.

El resultado positivo con la administración de 250 mg de enantato de testosterona vía intramuscular cada 4 semanas en este caso fue consistente con el observado en otros casos de síndrome Klinefelter asociados a úlceras en miembros pélvicos. En nuestro caso seleccionamos el método de administración intramuscular de testosterona depósito, ya que se sabe de su utilidad para producir concentraciones de testosterona en suero normales en 10 a 20 días posterior de la aplicación ${ }^{8}$. Sin embargo, tanto la asociación de úlceras en miembros inferiores con el síndrome de Klinefelter como la respuesta positiva a la terapia de testosterona son contradictorios respecto a los datos en animales que sugieren que la testosterona es perjudicial para la curación de heridas y que la inhibición de la actividad de la testosterona mejora la cicatrización de la herida ${ }^{9}$ (Figs. 1-4).

\section{CONCLUSIÓN}

Aunque ha pasado más de medio siglo desde la primera descripción del síndrome de Klinefelter, aún sigue siendo un desafío para el clínico. En el caso de este paciente, el tratamiento con testosterona se retrasó por varios años. Hay que insistir en la importancia de sospechar dicha patología en casos característicos como el descrito, cuyo tratamiento adecuado de manera oportuna hubiese 


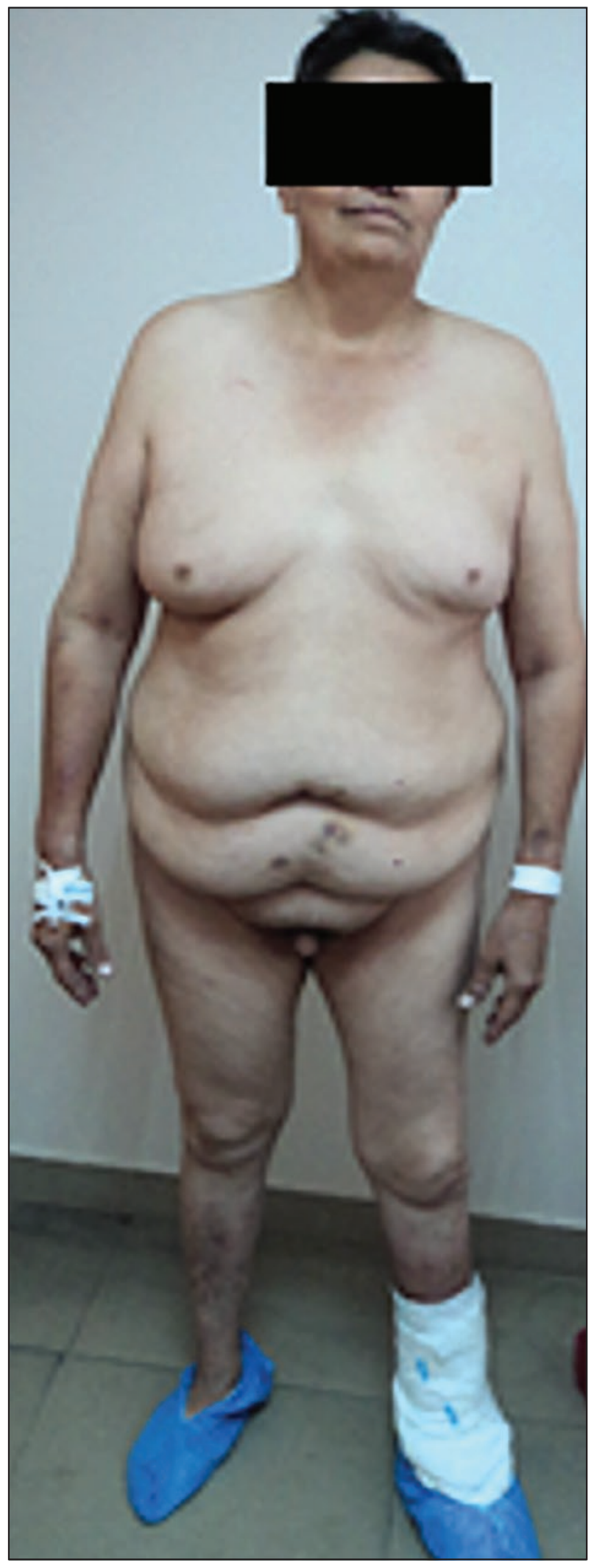

Figura 1. Fenotipo ginecoide.

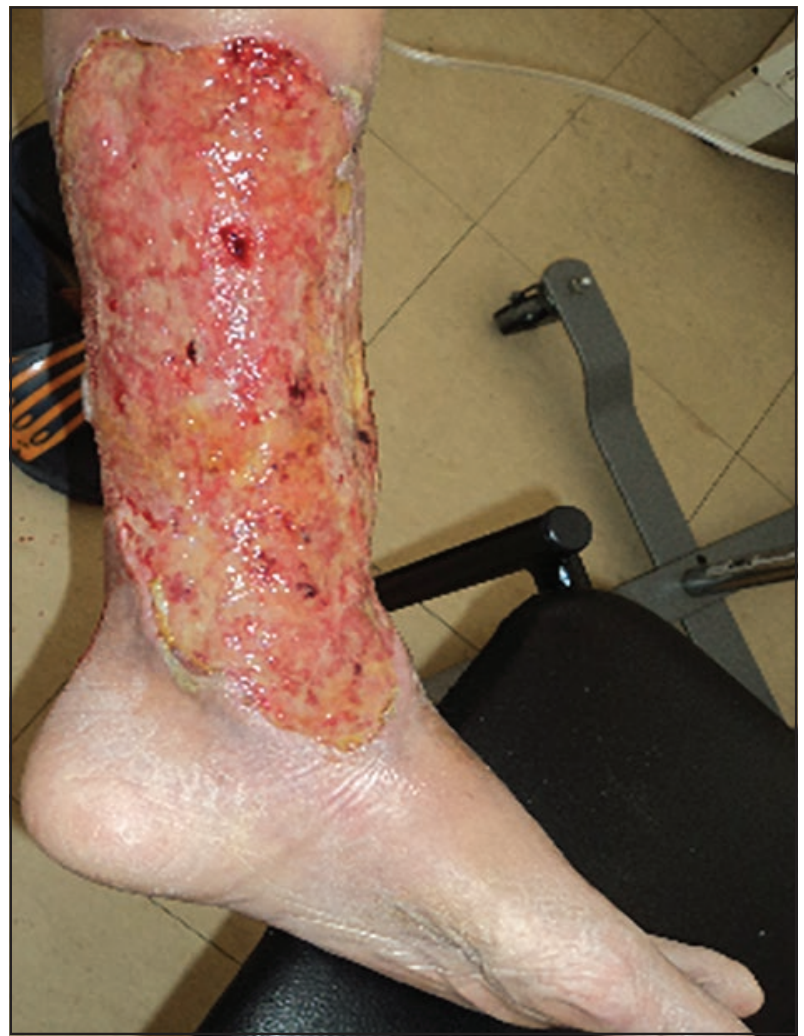

Figura 2. Cara interna de úlcera de pierna izquierda, en curaciones.

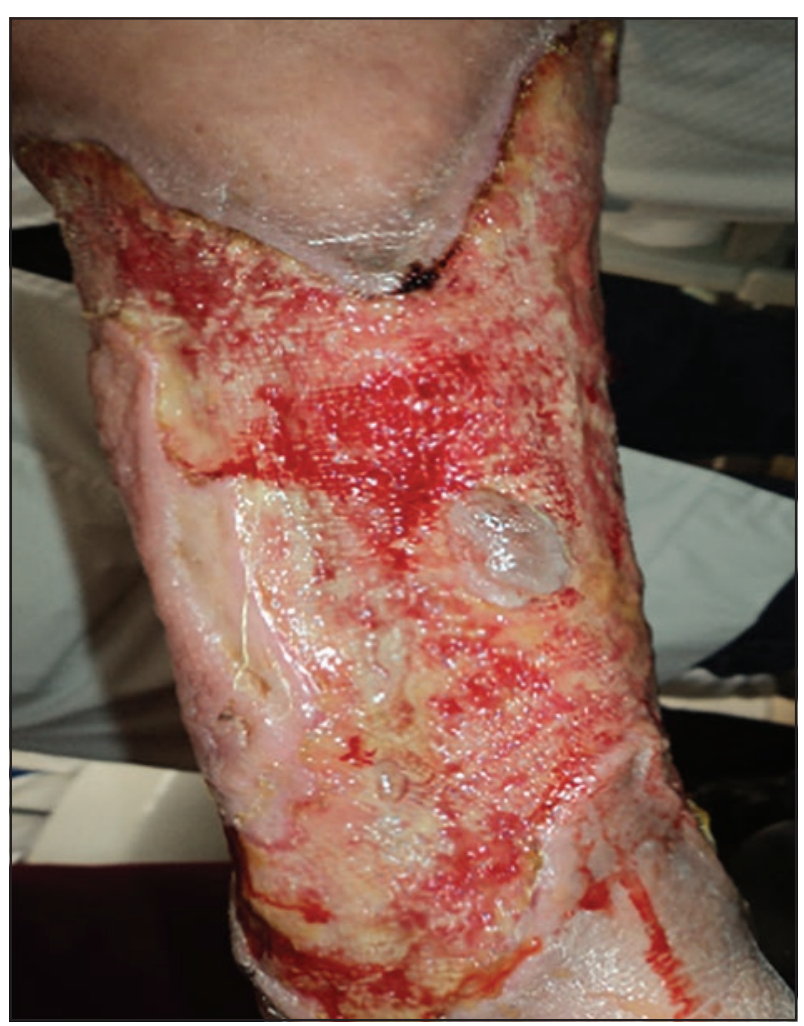

Figura 3. Cara posterior de úlcera de pierna izquierda, en curaciones. 


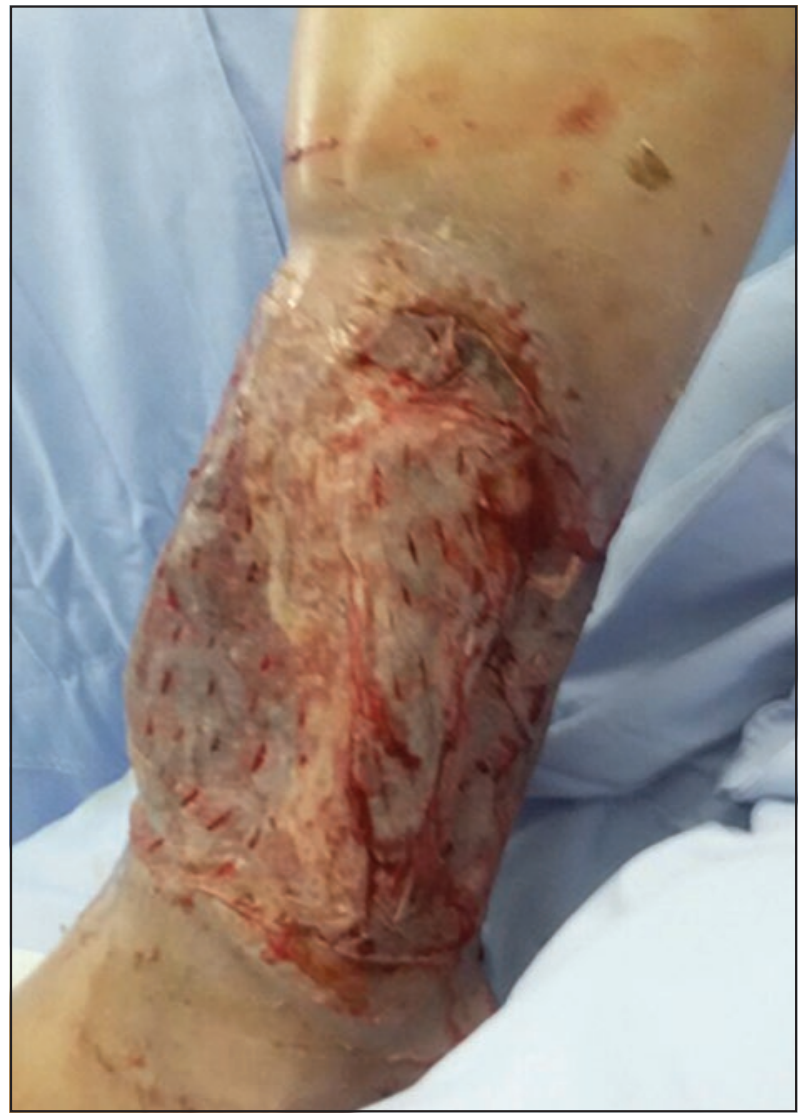

Figura 4. Úlcera crónica luego de la reposición hormonal con testosterona.

permitido la cicatrización de la úlcera en etapas tempranas.

Existe clara evidencia del avance de la tecnología en el laboratorio para la toma e interpretación del cariotipo, pero el tratamiento continúa siendo un reto y el pronóstico reservado.

\section{FINANCIAMIENTO}

Todas las fases de este estudio se realizaron con financiamiento por parte de los autores.

\section{CONFLICTO DE INTERESES}

Los autores declaran la no existencia de conflicto de intereses en la realización de la presente publicación.

\section{RESPONSABILIDADES ÉTICAS}

Protección de personas y animales. Los autores declaran que para esta investigación no se han realizado experimentos en seres humanos ni en animales.

Confidencialidad de los datos. Los autores declaran que han seguido los protocolos de su centro de trabajo sobre la publicación de datos de pacientes.

Derecho a la privacidad y consentimiento informado. Los autores han obtenido el consentimiento informado de los pacientes y/o sujetos referidos en el artículo. Este documento obra en poder del autor de correspondencia.

\section{BIBLIOGRAFÍA}

1. Bojesen A, Juul S, Gravholt $\mathrm{CH}$. Prenatal and postnatal prevalence of Klinefelter syndrome: a national registry study. J Clin Endocrinol Metab. 2003;88:6226.

2. Bojesen A, Gravholt CH. Klinefelter syndrome in clinical practice. Nat Clin Pract Urol. 2007;4:192204.

3. Bojesen A, Kristensen $\mathrm{K}$, Birkebaek NH, Fedder J, Mosekilde L, Bennett $\mathrm{P}$, et al. The metabolic syndrome is frequent in Klinefelter's syndrome and is associated with abdominal obesity and hypogonadism. Diabetes Care. 2006;29:15918.

4. Wikström A, Dunkel L. Klinefelter's syndrome. Best Pract Res Clin Endocrinol Metab. 2011;25:239-50.

5. Sarkar PK, Ballantyne S. Management of leg ulcers. Postgrad Med J 2000; 76(901):674-82.

6. Campbell W, Newton M, Price W. Hypostatic leg ulceration and Klinefelter's syndrome. J Ment Defic Res. 1980;24(2):115-7.

7. Swerdloff RS, Wang C, Cunningham G, Dobs A, Iranmanesh A, Matsumoto $A M$, et al. Long-term pharmacokinetics of transdermal testosterone gel in hypogonadal men. J Clin Endocrinol Metab. 2000;85:450010.

8. Gilliver SC, Ashworth JJ, Mills SJ, Hardman MJ, Ashcroft GS. Androgen modulate the inflammatory response during acute wound healing. J Cell Sci. 2006;119(Pt 4):72232.

9. Ashcroft GS, Mills SJ, Lei K, Gibbons L, Jeong MJ, Taniguchi M, et al. Estrogen modulates cutaneous wound healing by downregulating macrophage migration inhibitory factor. J Clin Invest. 2003;111:130918. 